Fábio Henrique Mendonça Corrêa (D) https://orcid.org/0000-0002-4591-205x

Bráulio Brandão Rodrigues² Ohttps://orcid.org/0000-0003-0860-9278

Jussane Cabral Mendonça' Ohttps://orcid. org/0000-0002-0509-1162

Leonardo Rodrigues da Cruz ${ }^{3}$ Dhttps://orcid.org/0000-0002-7429-9493

\section{Pensamento suicida entre a população transgênero: um estudo epidemiológico}

\author{
Suicide thoughts among transgender population: an epidemiological study
}

DOI: 10.1590/0047-2085000000256

\section{RESUMO}

Objetivo: Analisar a prevalência de pensamentos suicidas em pacientes de um ambulatório de transgêneros no Distrito Federal, bem como as variáveis associadas a esses eventos. Métodos: Estudo epidemiológico de corte transversal, realizado a partir de uma revisão dos prontuários dos pacientes atendidos no ambulatório de transgêneros do Hospital Dia, no Distrito Federal, no período de agosto de 2017 a setembro de 2018. Os dados foram tabulados pelo programa Excel 2016 e depois analisados pelo Pacote Estatístico para as Ciências Sociais (SPSS). Resultados: Neste estudo, observamos fatores intervenientes e variáveis que influenciam o desenvolvimento do comportamento suicida, com uma correlação entre fatores de risco modificáveis e não modificáveis. Os achados deste estudo corroboram a literatura internacional sobre comportamento suicida e as variáveis que podem estar relacionadas a esse fato. Conclusão: Questões sociodemográficas, de habitação e psiquiátricas compõem esses fatores, demonstrando possíveis relações com a ideação suicida. Assim, é possível mostrar situações de intervenção e elaboração de estratégias e políticas que possam melhorar a qualidade de vida desses indivíduos e reduzir as taxas de comportamento suicida.

\section{PALAVRAS-CHAVE}

Pessoas transgênero, identidade de gênero, disforia de gênero, serviços de saúde para pessoas transexuais, tentativa de suicídio.

\section{ABSTRACT}

Objective: To analyze the prevalence of suicidal thoughts among patients from a transgender outpatient clinic in the Federal District, as well as the variables associated with these events. Methods: A cross-sectional epidemiological study was carried out based on a review of the medical records of patients attending the transgender outpatient clinic of the Hospital Dia, in the Federal District, from August 2017 to September 2018. Data were then tabulated by the program Excel 2016 and then analyzed by the Statistical Package for the Social Sciences (SPSS). Results: In this study, we observed intervening and variable factors influencing the development of suicidal behavior, with a correlation between modifiable and non-modifiable risk factors. The findings of this study corroborate with the international literature on suicidal behavior, and the variables that may be related to this fact. Conclusion: Socio-demographic, housing, and psychiatric issues compose these factors, demonstrating possible relationships with suicidal ideation. Thus, it is possible to show situations of intervention and the elaboration of strategies and policies that can improve the quality of life of these individuals and reduce rates of suicidal behavior.

\section{KEYWORDS}

Transgender people, gender identity, gender dysphoria, health services for transgender people, suicide attempt.

Received in: Nov/22/2019. Approved in: 14/Dec/2019

1 Hospital São Vicente de Paulo, Brasília, DF, Brasil.

2 Centro Universitário de Anápolis, Anápolis, G0, Brasil.

3 Centro de Atenção Psicossocial - CAPS III Samambaia, Brasília, DF, Brasil.

Address for correspondence: Fábio Henrique Mendonça Corrêa. Telefone: (62) 98221-9241. E-mail: fabio.uni3@gmail.com 


\section{INTRODUÇÃO}

Quando se diz respeito a sexo, ele está relacionado com o órgão genital presente ao nascimento, podendo ser macho, fêmea ou hermafrodita. É por meio do sexo que se ocorre a reprodução humana. Em contrapartida, gênero está relacionado a uma construção social, psicológica e cultural, na qual o indivíduo se insere e se identifica, de acordo com determinadas características'. É importante salientar que o gênero não necessariamente está relacionado com o sexo biológico, inclusive vai muito além do feminino e masculino, considerado o binário, e muitos ainda nem estão registrados. Já a expressão de gênero é como o indivíduo vai se compor conforme sua identidade. Essa expressão é imposta pela sociedade, família, igreja, desde cedo na criança, quando se determina como cada sexo biológico deve se comportar².

A identidade de gênero pode ser caracterizada como a percepção do indivíduo sobre ser homem ou mulher, ou um gênero que foge a essa classificação, não binário, sendo sua expressão caracterizada como representação física dessa identificação. O gênero é uma categorização que tradicionalmente denomina homens ou mulheres a partir de aspectos físicos e características secundárias dos corpos masculinos e femininos ${ }^{3-5}$. Contudo, algumas pessoas não se enquadram em nenhum desses gêneros, distanciando-se do sexo de nascimento, aquele determinado pelo cariótipo $X X$ e $X Y^{5,6}$. Com isso, surge o conceito de transgeneridade, categoria que inclui pessoas transexuais e travestis. Tal designação é definida por pessoas na qual o sexo biológico e o gênero não estão alinhados. Algumas vezes também não se enquadram na categorização binária de gênero, sendo eles masculino e feminino ${ }^{7}$.

Esses indivíduos sofrem com preconceito, discriminação, sentimento de invisibilidade e violação de todas as naturezas promovidos pela sociedade no mundo atual. Tais condições dificultam ainda mais o acesso dos transexuais aos serviços públicos, entre eles educação, saúde, meios sociais e segurança pública. Eles apresentam ainda como agravantes de sua saúde riscos à integridade psíquica, física e moral, causando redução da qualidade de vida e promovendo danos à saúde mental e física principalmente, sendo os processos de sofrimento e sensação de inferioridade os principais exemplos ${ }^{8}$.

Nesse contexto, percebem-se impactos da transfobia na saúde mental e na privação de direitos de pessoas transexuais. Além disso, os indivíduos transexuais estão sujeitos a altos índices de discriminação social, sendo vítimas de vários tipos de violência, com maior predomínio da verbal. Em um estudo na população sueca, a prevalência de ideação suicida foi quase sete vezes e meia maior entre os entrevistados transexuais, e demonstrou-se também que o tratamento ofensivo e a violência são correlatos significativos da tendência suicida9. Em outro estudo, todos entrevistados relataram já ter sofrido algum tipo de violência ${ }^{10}$. Há uma conexão direta en- tre suicídio e saúde mental precária, particularmente doença mental. O suicídio e a automutilação são problemas graves entre minorias sexuais e tendem a ser determinados por um ambiente homofóbico e transfóbico, vários antecedentes da má saúde mental e fatores como o desemprego e dívidas, no contexto de um prolongado declínio econômico"1 ${ }^{11}$

A inconformidade com o gênero apresentou influência no comportamento suicida, predispondo à exposição deles a discriminação e a comportamentos lesivos à saúde. Eles tendem a relatar episódios de transfobia com maior frequência, sofrendo tratamento discriminatório, e com tendência ao abuso de drogas ilícitas, álcool e tabagismo. Os homens trans possuem inclinação a usar mais drogas ilícitas e a fumar mais que as mulheres, em decorrência da maior dificuldade de aceitação social. Além disso, os indivíduos com maiores rendas, níveis educacionais mais elevados e mais velhos são mais propensos a adquirir hábitos saudáveis do que suas contrapartes, com isso apresentam prevalências inferiores de comportamento suicida ${ }^{12}$.

Outro fator importante a ser ressaltado é em relação a raça, etnia e sofrimento psíquico, tendo em vista as minorias sociais, como a população negra e indígena. O preconceito decorre de representações sobre a diferença e o racismo aponta para a justificação e a racionalização de atitudes discriminatórias, de tal forma que o agressor poucas vezes reconhece a dimensão racista de seu discurso. Trata-se de indivíduos que julgam por atributos físicos e superficiais, expressos na identidade do afrodescendente, mas que direcionam a origem do racismo para a sociedade de forma genérica e imprecisa, ausentando-se de qualquer responsabilidade pelo tratamento discriminatório ${ }^{13}$.

Os transgêneros, especialmente os jovens, configuram-se como suscetíveis a piores condições de saúde mental, com predisposição a desenvolver depressão, ansiedade, autoflagelação e comportamento suicida, quando comparados à população cisgênera ${ }^{14}$. O Ambulatório de Assistência Especializada às Pessoas Travestis e Transexuais (Ambulatório Transgênero) se caracteriza como um serviço especializado no atendimento de pessoas com mais de 18 anos, com identidade de gênero destoante do sexo designado no nascimento, que procuram intervenções anatômicas, ou não, em processo de adaptação ao padrão de gênero identificado. Este trabalho tem como objetivo analisar a prevalência do pensamento suicida entre os pacientes de um ambulatório transgênero do Distrito Federal, assim como as variáveis associadas a esse comportamento.

\section{MÉTODOS}

Realizou-se um estudo transversal e observacional, a partir da revisão dos prontuários de pacientes atendidos no ambulatório transgênero do Hospital Dia, no Distrito Federal. 
Foi efetuado um levantamento de todos os prontuários dos pacientes atendidos no ambulatório voltado à população transexual, sendo aplicados os critérios de inclusão (ser maior de 18 anos; ter sido atendido pelo menos uma vez no Ambulatório Transgênero) e exclusão (pacientes não residentes no Distrito Federal e que se recusaram participar da pesquisa). Coletaram-se informações dos prontuários dos atendimentos ocorridos de agosto de 2017 a setembro de 2018.

O banco de dados foi construído a partir da transcrição dos dados obtidos no prontuário para o programa Excel 2016, obtido no Microsoft Office Access 2016. Os dados, em seguida, foram transferidos para o programa Statistical Package for the Social Sciences (SPSS Inc., Chicago, Estados Unidos). Foi aplicado o teste qui-quadrado, a fim de comparar as variáveis obtidas.

A análise do projeto de pesquisa foi executada mediante a Resolução n 466/2012 do CNS (Conselho Nacional de Saúde), na qual estão presentes as diretrizes e normas que regulamentam pesquisas que envolvam seres humanos. Essa resolução resguarda os referenciais básicos da bioética: beneficência, autonomia, justiça e não maleficência. Fundamentada nesses princípios, a pesquisa foi avaliada pelo Comitê de Ética de Pesquisa (CEP), a fim de ser outorgada. O presente estudo foi aprovado no CEP com CAAE: 02937018.5.0000.5553 e parecer n 3.137.865. Este trabalho é isento de conflito de interesses de acordo com as normas da Resolução no 1595/2000 do CFM e da RCD no 096/2008 da Agência Nacional de Vigilância Sanitária (Anvisa).

\section{RESULTADOS}

Foram analisados 221 prontuários, entre os quais, após aplicados os critérios de inclusão e exclusão, 194 mostraram-se aptos a esta pesquisa. Na análise dos dados epidemiológicos (Tabela 1), notou-se que os indivíduos com idade inferior a 20 anos (24,7\%) e entre 21 e 25 anos (32\%) predominaram sobre os demais. O mesmo ocorreu com relação a raça/cor parda $(47,4 \%)$, sexo de nascimento feminino $(58,2 \%)$, estado civil solteiro(a) (71,6\%), escolaridade com ensino médio completo $(27,8 \%)$ e superior incompleto $(29,4 \%)$. A maioria dos pacientes não possuía religião (57,7\%), estava indiferente $(36,6 \%)$ ou satisfeita $(23,7 \%)$ com a moradia, sendo essa predominantemente alugada $(41,8 \%)$.

No que tange à identidade de gênero e à expressão de gênero (Tabela 2), verifica-se um predomínio de homens transgênero $(56,2 \%)$, com a expressão de gênero do sexo masculino (56,7\%), e orientação sexual heterossexual (59,3\%). Cerca de $98,5 \%$ possuíam nome social. Sobre a aprovação familiar, houve predomínio de 27,3\% que são aprovados parcialmente e 26,8\% que são desaprovados. Em média, 50\% dos indivíduos já usaram algum recurso para modificar o corpo; desses, 50\% utilizaram hormônios e 10,3\% realizaram
Tabela 1. Distribuição por frequência simples dos dados socioeconômicos

\begin{tabular}{|c|c|c|}
\hline & & $f(\%)$ \\
\hline \multirow[t]{8}{*}{ Faixa etária } & $\leq 20$ & $48(24,7 \%)$ \\
\hline & $21-25$ & $62(32,0 \%)$ \\
\hline & $26-30$ & $39(20,1 \%)$ \\
\hline & $31-35$ & $16(8,2 \%)$ \\
\hline & $36-40$ & $11(5,7 \%)$ \\
\hline & $41-45$ & $10(5,2 \%)$ \\
\hline & $46-50$ & $3(1,5 \%)$ \\
\hline & $\geq 51$ & $5(2,6 \%)$ \\
\hline \multirow[t]{6}{*}{ Raça/cor } & Não respondeu & $2(1 \%)$ \\
\hline & Negro & $24(12,4 \%)$ \\
\hline & Pardo & $92(47,4 \%)$ \\
\hline & Branco & $68(35,1 \%)$ \\
\hline & Amarelo & $6(3,1 \%)$ \\
\hline & Indígena & $2(1 \%)$ \\
\hline \multirow[t]{2}{*}{ Sexo de nascimento } & Masculino & $81(41,8 \%)$ \\
\hline & Feminino & $113(58,2 \%)$ \\
\hline \multirow[t]{6}{*}{ Estado civil } & Não respondeu & $1(0,5 \%)$ \\
\hline & Casado/união estável & $34(17,5 \%)$ \\
\hline & Divorciado & $2(1 \%)$ \\
\hline & Solteiro & $139(71,6 \%)$ \\
\hline & Viúvo & $2(1 \%)$ \\
\hline & Não quer responder & $16(8,2 \%)$ \\
\hline \multirow[t]{9}{*}{ Escolaridade } & Não respondeu & $2(1 \%)$ \\
\hline & Ensino fundamental I & $3(1,5 \%)$ \\
\hline & Ensino fundamental II & $11(5,7 \%)$ \\
\hline & Ensino médio incompleto & $34(17,5 \%)$ \\
\hline & Ensino médio completo & $54(27,8 \%)$ \\
\hline & Ensino superior incompleto & $57(29,4 \%)$ \\
\hline & Ensino superior completo & $19(9,8 \%)$ \\
\hline & Curso técnico & $9(4,6 \%)$ \\
\hline & Pós-graduação & $5(2,6 \%)$ \\
\hline \multirow[t]{3}{*}{ Possui religião } & Não respondeu & $4(2,1 \%)$ \\
\hline & Sim & $78(40,2 \%)$ \\
\hline & Não & $112(57,7 \%)$ \\
\hline \multirow[t]{5}{*}{ Satisfação com moradia } & Muito insatisfeito & $18(9,3 \%)$ \\
\hline & Insatisfeito & $15(7,7 \%)$ \\
\hline & Indiferente & $71(36,6 \%)$ \\
\hline & Satisfeito & $46(23,7 \%)$ \\
\hline & Muito satisfeito & $44(22,7 \%)$ \\
\hline \multirow[t]{6}{*}{ Contexto da moradia } & Não respondeu & $46(23,7 \%)$ \\
\hline & Própria & $43(22,2 \%)$ \\
\hline & Alugada & $81(41,8 \%)$ \\
\hline & Financiada & $10(5,2 \%)$ \\
\hline & Cedida & $11(5,7 \%)$ \\
\hline & Invadida & $3(1,5 \%)$ \\
\hline
\end{tabular}

Dados obtidos por frequência simples com $n=194$ (100\%). 
cirurgia plástica. Cerca de 56,7\% tiveram acompanhamento psiquiátrico prévio, enquanto 66\% alegam não ter comorbidades psiquiátricas e 6,7\% possuíam internação psiquiátrica prévia. Em média, 73,7\% possuíam pensamentos suicidas em algum momento da vida e 29,9\% haviam tentado de fato pelo menos uma vez, além de 26,8\% já terem tentado automutilação/autoagressão, com predomínio de três ou mais episódios. A maioria dos pacientes apresentava sofrimento relacionado ao corpo (84\%), 67\% acharam que a vida valia a pena e $94,4 \%$ alegaram interesse em apoio especializado para poderem superar suas dificuldades/problemas.

Ao correlacionar o pensamento suicida com os fatores socioeconômicos (Tabela 3) por meio do teste qui-quadra- do de Pearson e razão de verossimilhança, notou-se que as faixas etárias $(p \leq 0,05)$ mais jovens estão mais associadas a esse quadro, com $79,2 \%$ dos membros com idade menor que 20 anos, 88,7\% entre aqueles com 21 a 25 anos e $71,8 \%$ dos com 26 a 30 anos. Em relação à cor/raça, os negros $(83,3 \%)$ e indígenas (100\%) são os que mais desenvolveram pensamentos suicidas. Além disso, o sexo de nascimento $(p \leq 0,05)$ feminino $(80,5 \%)$ apresentou maiores taxas dessa ideação que o sexo oposto. Na análise do estado civil $(p \leq 0,05)$, notou-se predomínio dos solteiros $(77,7 \%)$ no desenvolvimento dessa condição. Tem-se que aqueles com maior escolaridade $(p \leq 0,05)$ possuem maiores taxas de pensamento suicida, estando presente em $86 \%$ dos in-

Tabela 2. Distribuição por frequência simples dos dados referentes a questões acerca da sexualidade e histórico psiquiátrico

\begin{tabular}{|c|c|c|c|c|c|}
\hline & & $f(\%)$ & & & $f(\%)$ \\
\hline \multirow[t]{4}{*}{ Identidade de gênero } & Homem transgênero & $109(56,2 \%)$ & \multirow[t]{3}{*}{ Possui doença psiquiátrica? } & Não respondeu & $2(1 \%)$ \\
\hline & Mulher transgênero & $71(36,3 \%)$ & & Sim & $64(33 \%)$ \\
\hline & Travesti & $8(4,1 \%)$ & & Não & $128(66 \%)$ \\
\hline & Outros & $6(3,1 \%)$ & \multirow{3}{*}{$\begin{array}{l}\text { Internação psiquiátrica } \\
\text { prévia? }\end{array}$} & Não respondeu & $8(4,1 \%)$ \\
\hline \multirow[t]{3}{*}{ Expressão de gênero } & Masculino & $110(56,7 \%)$ & & Sim & $13(6,7 \%)$ \\
\hline & Feminino & $76(39,2 \%)$ & & Não & $173(89,2 \%)$ \\
\hline & Não binário & $8(4,1 \%)$ & \multirow[t]{3}{*}{ Pensamento suicida? } & Não respondeu & $10(5,2 \%)$ \\
\hline \multirow[t]{5}{*}{ Orientação sexual } & Não respondeu & $1(0,5 \%)$ & & $\operatorname{Sim}$ & $143(73,7 \%)$ \\
\hline & Homossexual & $21(10,8 \%)$ & & Não & $41(21,1 \%)$ \\
\hline & Heterossexual & $115(59,3 \%)$ & \multirow[t]{3}{*}{ Tentativa de suicídio? } & Não respondeu & $11(5,7 \%)$ \\
\hline & Bissexual & $56(28,9 \%)$ & & Sim & $58(29,9 \%)$ \\
\hline & Assexuado & $1(0,5 \%)$ & & Não & $125(64,4 \%)$ \\
\hline \multirow[t]{2}{*}{ Possui nome social? } & Não & $3(1,5 \%)$ & \multirow{6}{*}{$\begin{array}{l}\text { Quantas vezes tentou } \\
\text { suicídio? }\end{array}$} & Não tentou/respondeu & $137(70,6 \%)$ \\
\hline & Sim & $191(98,5 \%)$ & & 1 & $23(11,9 \%)$ \\
\hline \multirow[t]{6}{*}{ Aprovação familiar } & Não respondeu & $14(7,2 \%)$ & & 2 & $9(4,6 \%)$ \\
\hline & Aprova completamente & $30(15,5 \%)$ & & 3 & $23(11,9 \%)$ \\
\hline & Aprova parcialmente & $53(27,3 \%)$ & & 4 & $1(0,5 \%)$ \\
\hline & Indiferente & $25(12,9 \%)$ & & 6 & $1(0,5 \%)$ \\
\hline & Desaprova & $52(26,8 \%)$ & \multirow{3}{*}{$\begin{array}{l}\text { Tentou automutilação/ } \\
\text { autoagressão? }\end{array}$} & Não respondeu & $14(7,2 \%)$ \\
\hline & Não sabe & $20(10,3 \%)$ & & Sim & $52(26,8 \%)$ \\
\hline \multirow{4}{*}{$\begin{array}{l}\text { Utilizou recursos para } \\
\text { modificar o corpo? }\end{array}$} & Não respondeu & $8(4,1 \%)$ & & Não & $128(66 \%)$ \\
\hline & Sim & $97(50 \%)$ & \multirow{4}{*}{$\begin{array}{l}\text { Quantas vezes tentou } \\
\text { automutilação/ } \\
\text { autoagressão? }\end{array}$} & Não tentou/respondeu & $142(73,2 \%)$ \\
\hline & Não & 82 (42,3\%) & & 1 & $5(2,6 \%)$ \\
\hline & Não, mas gastaria & $7(3,6 \%)$ & & 2 & $2(1 \%)$ \\
\hline \multirow[t]{3}{*}{ Utilizou hormônios? } & Não respondeu & $7(3,6 \%)$ & & $\geq 3$ & $45(23,2 \%)$ \\
\hline & Sim & $97(50 \%)$ & \multirow{2}{*}{$\begin{array}{l}\text { Apresenta sofrimento } \\
\text { relacionado ao corpo? }\end{array}$} & Sim & $163(84 \%)$ \\
\hline & Não & $90(46,4 \%)$ & & Não & $31(16 \%)$ \\
\hline \multirow[t]{3}{*}{ Realizou cirurgia plástica? } & Não respondeu & $7(3,6 \%)$ & \multirow{2}{*}{$\begin{array}{l}\text { Acha que a vida vale a } \\
\text { pena? }\end{array}$} & Sim & $130(67 \%)$ \\
\hline & Sim & $20(10,3 \%)$ & & Não & $64(33 \%)$ \\
\hline & Não & $167(86,1 \%)$ & \multirow{2}{*}{$\begin{array}{l}\text { Gostaria de ajuda } \\
\text { especializada? }\end{array}$} & Sim & $183(94,3 \%)$ \\
\hline Acompanhamento & Não respondeu & $3(1,5 \%)$ & & Não & $11(5,7 \%)$ \\
\hline
\end{tabular}

$\begin{array}{lr}\text { Sim } & 110(56,7 \%) \\ \text { Não } & 81(41,8 \%)\end{array}$

Dados obtidos por frequência simples com n = 194 (100\%). 
divíduos com ensino superior incompleto, 78,9\% daqueles com ensino superior completo, $77,8 \%$ dos com curso técnico e $80 \%$ dos pós-graduandos. Tem-se ainda que os que não possuem vínculo religioso $(p \leq 0,05)$ possuem maiores índices de ideação suicida (82,1\%).

Apesar de os homens transexuais serem mais afetados com pensamento suicida, não houve significância estatística nessa correlação (Tabela 4). Os indivíduos que expressam gênero masculino apresentaram maior frequência de pensamento suicida (80\%). Sobre a orientação sexual ( $p \leq 0,05)$, notou-se que $72,2 \%$ dos heterossexuais e $87,5 \%$ dos bisse- xuais foram afetados por pensamentos suicidas. No quesito aprovação familiar $(p \leq 0,05)$, o fato de a família desaprovar ou desconhecer a orientação sexual dos indivíduos mostrou que, em ambos os casos, a frequência de ideação suicida pode atingir aproximadamente $90 \%$ dessa população. Além disso quando indagados sobre a utilização de recursos para modificar o corpo $(p \leq 0,05)$, aqueles que não realizaram intervenções $(80,5 \%)$ exibiram maior correlação com pensamentos de autoextermínio. A análise da influência do nome social, apesar de possuir significância estatística, é de difícil avaliação pois apenas 3 (1,5\%) participantes não o possuíam.

Tabela 3. Relação entre o pensamento suicida e os fatores socioeconômicos

\begin{tabular}{|c|c|c|c|c|c|c|}
\hline & & \multicolumn{3}{|c|}{ Pensamento suicida } & \multirow{2}{*}{ Total } & \multirow{2}{*}{ Significância } \\
\hline & & Não respondeu & Sim & Não & & \\
\hline \multirow[t]{8}{*}{ Faixa etária } & $\leq 20$ anos & 0 & $38(79,2 \%)$ & $10(20,8 \%)$ & $48(100 \%)$ & $p \leq 0,05$ \\
\hline & 21 a 25 anos & 0 & $55(88,7 \%)$ & $7(11,3 \%)$ & $62(100 \%)$ & \\
\hline & 26 a 30 anos & $1(2,6 \%)$ & $28(71,8 \%)$ & $10(25,6 \%)$ & $39(100 \%)$ & \\
\hline & 31 a 35 anos & $1(6,3 \%)$ & $9(56,3 \%)$ & $6(37,5 \%)$ & $16(100 \%)$ & \\
\hline & 36 a 40 anos & $4(36,4 \%)$ & $4(36,4 \%)$ & $3(27,3 \%)$ & $11(100 \%)$ & \\
\hline & 41 a 45 anos & $1(10 \%)$ & $7(70 \%)$ & $2(20 \%)$ & $10(100 \%)$ & \\
\hline & 46 a 50 anos & $1(33,3 \%)$ & $1(33,3 \%)$ & $1(33,3 \%)$ & $3(100 \%)$ & \\
\hline & $>50$ anos & $2(40 \%)$ & $1(20 \%)$ & $2(40 \%)$ & $5(100 \%)$ & \\
\hline \multirow[t]{6}{*}{ Cor/raça } & Não respondeu & 0 & $2(100 \%)$ & 0 & $2(100 \%)$ & $p=0,56$ \\
\hline & Negro & 0 & $20(83,3 \%)$ & $4(16,7 \%)$ & $24(100 \%)$ & \\
\hline & Pardo & $7(7,6 \%)$ & $63(68,5 \%)$ & $22(23,9 \%)$ & $92(100 \%)$ & \\
\hline & Branco & $3(4,4 \%)$ & $53(77,9 \%)$ & $12(17,6 \%)$ & $68(100 \%)$ & \\
\hline & Amarelo & 0 & $3(50 \%)$ & $3(50 \%)$ & $6(100 \%)$ & \\
\hline & Indígena & 0 & $2(100 \%)$ & 0 & $2(100 \%)$ & \\
\hline \multirow{2}{*}{$\begin{array}{l}\text { Sexo de } \\
\text { nascimento }\end{array}$} & Masculino & $7(8,6 \%)$ & $52(64,2 \%)$ & $22(27,2 \%)$ & $81(100 \%)$ & $p \leq 0,05$ \\
\hline & Feminino & $3(2,7 \%)$ & $91(80,5 \%)$ & $19(16,8 \%)$ & $113(100 \%)$ & \\
\hline \multirow[t]{5}{*}{ Estado civil } & Não respondeu & $1(5,9 \%)$ & $10(58,9 \%)$ & $6(35,2 \%$ & $17(100 \%)$ & $p \leq 0,05$ \\
\hline & Casado/união estável & $2(5,9 \%)$ & $24(70,6 \%)$ & $8(23,5 \%)$ & $34(100 \%)$ & \\
\hline & Divorciado & $1(50 \%)$ & $1(50 \%)$ & 0 & $2(100 \%)$ & \\
\hline & Solteiro & $5(3,6 \%)$ & $108(77,7 \%)$ & $26(18,7 \%)$ & $139(100 \%)$ & \\
\hline & Viúvo & $1(50 \%)$ & 0 & $1(50 \%)$ & $2(100 \%)$ & \\
\hline \multirow[t]{9}{*}{ Escolaridade } & Não respondeu & 0 & $1(50 \%)$ & $1(50 \%)$ & $2(100 \%)$ & $p \leq 0,05^{\star}$ \\
\hline & Ensino Fundamental I & $1(33,3 \%)$ & 0 & $2(66,7 \%)$ & $3(100 \%)$ & \\
\hline & Ensino Fundamental II & $1(9,1 \%)$ & $8(72,2 \%)$ & $2(18,2 \%)$ & $11(100 \%)$ & \\
\hline & Ensino médio incompleto & $2(5,9 \%)$ & $24(70,6 \%)$ & $8(23,5 \%)$ & $34(100 \%)$ & \\
\hline & Ensino médio completo & $5(9,3 \%)$ & $35(64,8 \%)$ & $14(25,9 \%)$ & $54(100 \%)$ & \\
\hline & Ensino superior incompleto & 0 & $49(86 \%)$ & $8(14 \%)$ & $57(100 \%)$ & \\
\hline & Ensino superior completo & 0 & $15(78,9 \%)$ & $4(21,4 \%)$ & $19(100 \%)$ & \\
\hline & Curso técnico & 0 & $7(77,8 \%)$ & $2(22,2 \%)$ & $9(100 \%)$ & \\
\hline & Pós-graduação & $1(20 \%)$ & $4(80 \%)$ & 0 & $5(100 \%)$ & \\
\hline \multirow[t]{3}{*}{ Religião } & & $2(50 \%)$ & $1(25 \%)$ & $1(25 \%)$ & $4(100 \%)$ & $p \leq 0,05$ \\
\hline & Possui & $5(6,4 \%)$ & $50(64,1 \%)$ & $23(29,5 \%)$ & $78(100 \%)$ & \\
\hline & Não possui & $3(2,7 \%)$ & $92(82,1 \%)$ & 17 (15,2\%) & 112 (100\%) & \\
\hline
\end{tabular}

Dados fornecidos com $n(\%)$. Foi realizado teste qui-quadrado de Pearson, tendo $p \leq 0,05$ como valor de significância estatística. * Foi analisado a partir de teste qui-quadrado por razão de verossimilhança. 
A relação entre o pensamento suicida e comorbidades psiquiátricas (Tabela 5) evidenciou que o grupo dos pacientes em acompanhamento psiquiátrico $(p \leq 0,05)$ desenvolveu mais pensamentos suicidas (77,3\%), assim como o daqueles com doença psiquiátrica $(p \leq 0,05)$ autorreferida (87,5\%), e os com histórico positivo para internações prévias $(p \leq 0,05)$ pela psiquiatria $(92,3 \%)$. Observou-se $(p \leq 0,05)$ que todos os integrantes com pensamentos suicidas prévios tentaram autoextermínio, diferentemente daqueles (68\%) que não apresentaram ideação prévia. Quando abordados sobre a quantidade de tentativas ( $p \leq 0,05), 58,1 \%$ dos pacientes que relataram tentativas prévias não especificaram quantas vezes o fizeram, e os demais tenderam a ter mais de um episódio. Todos os pacientes com um a dois episódios de autoagressão/mutilação apresentavam pensamentos suicidas, sendo esses encontrados em 93,3\% dos que apresentaram no mínimo três episódios autolesivos $(p<0,05)$. Além disso, também foi notada uma relação positiva $(p \leq 0,05)$ entre $o$ pensamento suicida e os grupos dos pacientes com sofrimento com o corpo (77,9\%), sentimento que a vida não vale a pena $(79,7 \%)$ e busca por ajuda especializada $(75,4 \%)$. A relação entre as comorbidades psiquiátricas e o pensamento suicida evidenciou-se estatisticamente significativa entre os indivíduos que autorreferiram ansiedade (82,4\%), depressão $(92,3 \%)$ e as duas simultaneamente (100\%).

\section{DISCUSSÃO}

Este estudo constatou que os pacientes atendidos no ambulatório transgênero enfrentam iniquidades em saúde, incluindo consequências graves, como aumento da probabilidade de suicídio. Semelhante aos achados de outros estudos, a taxa de suicídio entre as pessoas transexuais é desproporcionalmente alta.

Em relato com 154 participantes transexuais, foi observado que $48,3 \%$ possuíam ideação suicida e $23,8 \%$ tentaram suicídio, constatando que existem níveis mais altos de ideação e tentativas suicidas em pessoas com disforia de gênero do que na população geral. Eles não observaram diferenças

Tabela 4. Relação entre o pensamento suicida e as questões de sexualidade e gênero

\begin{tabular}{|c|c|c|c|c|c|c|}
\hline & & \multicolumn{3}{|c|}{ Pensamento suicida } & \multirow{2}{*}{ Total } & \multirow{2}{*}{ Significância } \\
\hline & & Não respondeu & Sim & Não & & \\
\hline \multirow{4}{*}{$\begin{array}{l}\text { Identidade de } \\
\text { gênero }\end{array}$} & Homem transgênero & $3(2,80 \%)$ & $87(79,80 \%)$ & $19(17,40 \%)$ & $109(100,00 \%)$ & \multirow[t]{4}{*}{0,085} \\
\hline & Mulher transgênero & $7(9,90 \%)$ & $44(62,00 \%)$ & $20(28,20 \%)$ & $71(100,00 \%)$ & \\
\hline & Travesti & $0(0,00 \%)$ & $6(75,00 \%)$ & $2(25,00 \%)$ & $8(100,00 \%)$ & \\
\hline & Outros & $0(0,00 \%)$ & $6(100,00 \%)$ & $0(0,00 \%)$ & $6(100,00 \%)$ & \\
\hline \multirow{3}{*}{$\begin{array}{l}\text { Expressão de } \\
\text { gênero }\end{array}$} & Masculino & $3(2,70 \%)$ & $88(80,00 \%)$ & $19(17,30 \%)$ & $110(100,00 \%)$ & \multirow[t]{3}{*}{$p \leq 0,05$} \\
\hline & Feminino & $7(9,20 \%)$ & $47(61,80 \%)$ & $22(28,90 \%)$ & $76(100,00 \%)$ & \\
\hline & Não binário & $0(0,00 \%)$ & $8(100,00 \%)$ & $0(0,00 \%)$ & $8(100,00 \%)$ & \\
\hline \multirow{5}{*}{$\begin{array}{l}\text { Orientação } \\
\text { sexual }\end{array}$} & Não respondeu & $0(0,00 \%)$ & $0(0,00 \%)$ & $1(100,00 \%)$ & $1(100,00 \%)$ & \multirow[t]{5}{*}{$p \leq 0,05$} \\
\hline & Homossexual & $4(19,00 \%)$ & $10(47,60 \%)$ & $7(33,30 \%)$ & $21(100,00 \%)$ & \\
\hline & Heterossexual & $3(2,60 \%)$ & $83(72,20 \%)$ & $29(25,20 \%)$ & $115(100,00 \%)$ & \\
\hline & Bissexual & $3(5,40 \%)$ & $49(87,50 \%)$ & $4(7,10 \%)$ & $56(100,00 \%)$ & \\
\hline & Assexuado & $0(0,00 \%)$ & $1(100,00 \%)$ & $0(0,00 \%)$ & $1(100,00 \%)$ & \\
\hline \multirow{6}{*}{$\begin{array}{l}\text { Aprovação } \\
\text { familiar }\end{array}$} & Não respondeu & $8(57,10 \%)$ & $5(35,70 \%)$ & $1(7,10 \%)$ & $14(100,00 \%)$ & \multirow[t]{6}{*}{$p \leq 0,05$} \\
\hline & Aprova completamente & $1(3,30 \%)$ & $21(70,00 \%)$ & $8(26,70 \%)$ & $30(100,00 \%)$ & \\
\hline & Aprova parcialmente & $1(1,90 \%)$ & $33(62,30 \%)$ & $19(35,80 \%)$ & $53(100,00 \%)$ & \\
\hline & Indiferente & $0(0,00 \%)$ & 19 (76,00\%) & $6(24,00 \%)$ & $25(100,00 \%)$ & \\
\hline & Desaprova & $0(0,00 \%)$ & $47(90,40 \%)$ & $5(9,60 \%)$ & $52(100,00 \%)$ & \\
\hline & Não sabe & $0(0,00 \%)$ & $18(90,00 \%)$ & $2(10,00 \%)$ & $20(100,00 \%)$ & \\
\hline \multirow{4}{*}{$\begin{array}{l}\text { Utilizou recursos } \\
\text { para modificar } 0 \\
\text { corpo? }\end{array}$} & Não respondeu & $7(87,50 \%)$ & $1(12,50 \%)$ & $0(0,00 \%)$ & $8(100,00 \%)$ & \multirow[t]{4}{*}{$p \leq 0,05$} \\
\hline & Sim & $1(1,00 \%)$ & $72(74,20 \%)$ & $24(24,70 \%)$ & $97(100,00 \%)$ & \\
\hline & Não & $0(0,00 \%)$ & $66(80,50 \%)$ & $16(19,50 \%)$ & $82(100,00 \%)$ & \\
\hline & Não, mas gostaria & $2(28,60 \%)$ & $4(57,10 \%)$ & $1(14,30 \%)$ & $7(100,00 \%)$ & \\
\hline \multirow{2}{*}{$\begin{array}{l}\text { Possui nome } \\
\text { social? }\end{array}$} & Não & $2(66,70 \%)$ & $1(33,30 \%)$ & $0(0,00 \%)$ & $3(100,00 \%)$ & \multirow[t]{2}{*}{$p \leq 0,05$} \\
\hline & $\operatorname{Sim}$ & $8(4,20 \%)$ & $142(74,30 \%)$ & $41(21,50 \%)$ & $191(100,00 \%)$ & \\
\hline
\end{tabular}

Dados fornecidos com $n$ (\%). Foi realizado teste qui-quadrado de Pearson, tendo $p \leq 0,05$ como valor de significância estatística. 
entre os grupos em termos de sexo, e a morbidade psiquiátrica não foi uma variável importante para o comportamento suicida, sugerindo que a ideação suicida é um dos melhores indicadores do risco de comportamentos suicidas ${ }^{15}$. No presente estudo, houve concordância com os achados de prevalência maiores tanto para ideação como tentativa prévia de suicídio, assim como se notou relação do sexo de nascimento, gênero e orientação sexual com essas morbidades.

Em investigação realizada com uma população sueca, a prevalência de ideação suicida foi quase sete vezes e meia maior entre os entrevistados transexuais, e demonstrou-se também que o tratamento ofensivo e a violência são cor-

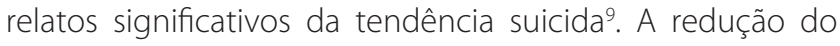
estigma e a mitigação de vulnerabilidades estruturais, por exemplo, a promulgação e a aplicação de leis que proíbem a discriminação baseada na identidade de gênero para garantir acesso equitativo à moradia, podem ser alvos efetivos de intervenção para reduzir as tentativas de suicídio entre indivíduos transgêneros ${ }^{16}$.
Um estudo norte-americano mostrou que enquanto a tentativa de suicídio na população geral de estadunidenses era de 1,6\%, nos indivíduos transgêneros entrevistados chegou a 41\%. De acordo com o Instituto Nacional de Saúde Mental (NIMH), grande parte dos casos ocorre com pessoas em situação de enorme aflição, e a população estudada se encontra em tal circunstância em diferentes campos da vida, devido à discriminação ainda existente, como a perda do emprego, por ser expulso de casa pela família e o abandono dos amigos ${ }^{17}$.

A incidência global de tentativa de suicídio entre os participantes de sua pesquisa foi de $29,8 \%(n=42)$; no total, 76,7\% das tentativas de suicídio ocorreram antes dos 21 anos ${ }^{15}$. Diversos estudos constataram pelo menos uma tentativa de suicídio ao longo da vida dos entrevistados, entre os quais a idade mediana na primeira tentativa foi entre $17 \mathrm{e}$ 21 anos ${ }^{11,15,16,18-20}$. Tal fato corrobora os achados do presente estudo, em que as maiores taxas de tentativa suicida ocorreram em idades mais precoces.

Tabela 5. Relação entre o pensamento suicida e comorbidades psiquiátricas

\begin{tabular}{|c|c|c|c|c|c|c|}
\hline & & \multicolumn{3}{|c|}{ Pensamento suicida } & \multirow{2}{*}{ Total } & \multirow{2}{*}{ Significância } \\
\hline & & Não respondeu & Sim & Não & & \\
\hline \multirow{3}{*}{$\begin{array}{l}\text { Acompanhamento } \\
\text { psiquiátrico anterior? }\end{array}$} & Não respondeu & $3(100,00 \%)$ & $0(0,00 \%)$ & $0(0,00 \%)$ & $3(100,00 \%)$ & $p \leq 0,05$ \\
\hline & $\operatorname{Sim}$ & $4(3,60 \%)$ & $85(77,30 \%)$ & $21(19,10 \%)$ & $110(100,00 \%)$ & \\
\hline & Não & $3(3,70 \%)$ & $58(71,60 \%)$ & $20(24,70 \%)$ & $81(100,00 \%)$ & \\
\hline \multirow{3}{*}{$\begin{array}{l}\text { Possui doença } \\
\text { psiquiátrica? }\end{array}$} & Não respondeu & $2(100,00 \%)$ & $0(0,00 \%)$ & $0(0,00 \%)$ & $2(100,00 \%)$ & $p \leq 0,05$ \\
\hline & $\operatorname{Sim}$ & $3(4,70 \%)$ & $56(87,50 \%)$ & $5(7,80 \%)$ & $64(100,00 \%)$ & \\
\hline & Não & $5(3,90 \%)$ & $87(68,00 \%)$ & 36 28,10\%) & $128(100,00 \%)$ & \\
\hline \multirow{3}{*}{$\begin{array}{l}\text { Internação psiquiátrica } \\
\text { prévia? }\end{array}$} & Não respondeu & $8(100,00 \%)$ & $0(0,00 \%)$ & $0(0,00 \%)$ & $8(100,00 \%)$ & $p \leq 0,05$ \\
\hline & $\operatorname{Sim}$ & $0(0,00 \%)$ & $12(92,30 \%)$ & $1(7,70 \%)$ & $13(100,00 \%)$ & \\
\hline & Não & $2(1,20 \%)$ & $131(75,70 \%)$ & $40(23,10 \%)$ & $173(100,00 \%)$ & \\
\hline \multirow[t]{3}{*}{ Tentativa de suicídio? } & Não respondeu & $10(90,90 \%)$ & $0(0,00 \%)$ & $1(9,10 \%)$ & $11(100,00 \%)$ & $p \leq 0,05$ \\
\hline & $\operatorname{Sim}$ & $0(0,00 \%)$ & $58(100,00 \%)$ & $0(0,00 \%)$ & $58(100,00 \%)$ & \\
\hline & Não & $0(0,00 \%)$ & $85(68,00 \%)$ & $40(32,00 \%)$ & $125(100,00 \%)$ & \\
\hline \multirow{4}{*}{$\begin{array}{l}\text { Quantas vezes tentou } \\
\text { suicídio? }\end{array}$} & Não respondeu & $10(7,30 \%)$ & $86(62,80 \%)$ & $41(29,90 \%)$ & $137(100,00 \%)$ & $p \leq 0,05$ \\
\hline & $1 \mathrm{vez}$ & $0(0,00 \%)$ & $23(100,00 \%)$ & $0(0,00 \%)$ & $23(100,00 \%)$ & \\
\hline & 2 vezes & $0(0,00 \%)$ & $9(100,00 \%)$ & $0(0,00 \%)$ & $9(100,00 \%)$ & \\
\hline & $\geq 3$ vezes & $0(0,00 \%)$ & $25(100,00 \%)$ & $0(0,00 \%)$ & $25(100,00 \%)$ & \\
\hline \multirow{4}{*}{$\begin{array}{l}\text { Tentou automutilação/ } \\
\text { autoagressão? }\end{array}$} & Não respondeu & $10(7,00 \%)$ & $94(66,20 \%)$ & $38(26,80 \%)$ & $142(100,00 \%)$ & $p \leq 0,05$ \\
\hline & $1 \mathrm{vez}$ & $0(0,00 \%)$ & $5(100,00 \%)$ & $0(0,00 \%)$ & $5(100,00 \%)$ & \\
\hline & 2 vezes & $0(0,00 \%)$ & $2(100,00 \%)$ & $0(0,00 \%)$ & $2(100,00 \%)$ & \\
\hline & $\geq 3$ vezes & $0(0,00 \%)$ & $42(93,30 \%)$ & $3(6,70 \%)$ & 45 (100,00\%) & \\
\hline \multirow{2}{*}{$\begin{array}{l}\text { Apresenta sofrimento } \\
\text { relacionado ao corpo? }\end{array}$} & $\operatorname{Sim}$ & $6(3,70 \%)$ & $127(77,90 \%)$ & $30(18,40 \%)$ & $163(100,00 \%)$ & $p \leq 0,05$ \\
\hline & Não & $4(12.90 \%)$ & $16(51,60 \%)$ & $11(35,50 \%)$ & $31(100,00 \%)$ & \\
\hline \multirow{2}{*}{$\begin{array}{l}\text { Acha que a vida vale a } \\
\text { pena? }\end{array}$} & Sim & $6(4,60 \%)$ & $92(70,80 \%)$ & $32(24,60 \%)$ & $130(100,00 \%)$ & $p \leq 0,05$ \\
\hline & Não & $4(6,30 \%)$ & $51(79,70 \%)$ & $9(14,10 \%)$ & $64(100,00 \%)$ & \\
\hline
\end{tabular}

Dados fornecidos com n (\%). Foi realizado teste qui-quadrado de Pearson, tendo $p \leq 0,05$ como valor de significância estatística. 
É importante ressaltar a questão do estado civil. Na literatura, o estado civil casado é apontado como um fator de proteção. Não se questiona a amplitude dos reflexos da realização do processo transexualizador, tanto na esfera particular do indivíduo quanto nas relações sociais que ele estabelece, principalmente no âmbito familiar. Contudo, devido ao preconceito e à discriminação, é muito difícil para uma pessoa transexual conseguir uma relação afetiva/amorosa ou se manter nela. Em vista disso, o estado civil de pessoas que apresentaram o comportamento suicida pode ser composto por um número elevado de sujeitos solteiros ${ }^{21,22}$.

A prevalência de tentativas de suicídio ao longo da vida, em vários países, é de 4\% para os entrevistados heterossexuais em inquéritos populacionais, 11\% para os participantes LGB em inquéritos feitos à população e $20 \%$ para os participantes $L G B$ de inquéritos comunitários ${ }^{23}$. Um estudo brasileiro mostrou que os indivíduos bissexuais são os que apresentam maiores índices de doenças mentais, entre elas ideação/tentativa de autoextermínio, principalmente os homens autodeclarados. Isso ocorre devido à discriminação que ocorre tanto pelos heterossexuais como pelos homossexuais. Dessa forma, o estado emocional encontra-se pior, assim como a sensação de isolamento se faz maior ${ }^{24}$.

Os indivíduos transexuais estão sujeitos a altos índices de discriminação social, sendo vítimas de vários tipos de violência, com maior predomínio da verbal. Em estudo com 316 participantes LGBT, todos os entrevistados relataram já ter sofrido algum tipo de violência. O consumo de drogas ocorreu em $84 \%$ e não houve diferença estatisticamente significante entre o tipo de violência sofrida e o tipo de droga utilizada ${ }^{25}$. Os níveis mais baixos de estigma estrutural foram associados a menos tentativas de suicídio, e um escore maior na escala de transfobia internalizada foi associado a tentativas de suicídio maiores ${ }^{10}$. A escolaridade se mostrou como fator de proteção para as tentativas de suicídio e automutilação/autoagressão, pois aqueles com nível superior de ensino apresentaram menores taxas desses eventos ${ }^{10,20,26,27}$. Tal dado contrasta com o observado nesse estudo, pois foram observados maiores valores para pensamento suicida e automutilação/autoagressão entre os indivíduos com maior grau de instrução.

Em análise com uma amostra de 7.758 alunos nas 9a e $11^{\text {a }}$ séries, tendo em vista que aproximadamente $4 \%$ deles eram considerados gays, lésbicas ou bissexuais, a conectividade dos pais apareceu como um fator de proteção robusto para todos os comportamentos de autoflagelação em todos os grupos sexuais. Sentir-se ligado a adultos e seguro na escola representou fator adicional que reduziu o risco ${ }^{28}$. O apoio dos pais para a identidade de gênero foi associado à redução da ideação ${ }^{29}$. Conforme relatado nesse levantamento, a falta de aprovação familiar ou o desconhecimento da família acerca das questões referentes à sexualidade desses indivíduos está relacionada ao aumento de ideação suicida e automutilação/autoagressão.
Há uma conexão direta entre suicídio e saúde mental precária, particularmente doença mental. O suicídio e a automutilação são problemas graves entre minorias sexuais e tendem a ser determinados por um ambiente homofóbico e transfóbico, vários antecedentes da má saúde mental e fatores como o desemprego e dívidas, no contexto de um prolongado declínio econômico ${ }^{11}$.

Estudo utilizando o prontuário de 180 pacientes transexuais (semelhante ao do trabalho atual) entre 12 e 29 anos atendidos em um centro comunitário demonstrou que os jovens transgênero tiveram um risco duplo ou triplicado de depressão, transtorno de ansiedade, ideação suicida, tentativa de suicídio e autoflagelação sem intenção letal. Constatou-se que os jovens transexuais têm uma desigualdade nos desfechos de saúde mental negativos em comparação com os jovens cisgêneros, por isso identificar as diferenças de identidade de gênero nos ambientes clínicos e fornecer serviços e apoios apropriados são passos importantes para abordar essa disparidade ${ }^{14}$.

Diversos estudos relataram a relação de doenças psiquiátricas e as taxas de ideação suicida e automutilação/ autoagressão, e a depressão e a ansiedade foram os principais diagnósticos encontrados ${ }^{15,19,28,30}$. A depressão configura um fator de risco para o comportamento suicida. Tais dados foram evidenciados na presente pesquisa, acrescidos ainda da associação entre ansiedade e depressão, que promovem juntas um possível acréscimo na tendência suicida ${ }^{19}$.

\section{CONCLUSÃO}

A saúde da população transgênero está relacionada a vulnerabilidades, tanto por características próprias de cada indivíduo quanto pelos determinantes da sociedade na qual estão inseridos. Há níveis extensos e amplamente variáveis de envolvimento em comportamentos de risco à saúde dentro da comunidade LGBT. Além disso, as subcategorias de gênero e orientação sexual, que são tradicionalmente condensadas em uma categoria (ou seja, transgênero e bissexual), evidenciaram riscos surpreendentemente diferentes quando examinadas separadamente.

Foram observados neste estudo fatores intervenientes e variáveis com influência no desenvolvimento do comportamento suicida, com uma correlação entre fatores de risco modificáveis e não modificáveis. As questões sociodemográficas, de moradia e psiquiátricas compõem esses fatores, demonstrando possíveis relações com o quadro de ideação suicida e automutilação/autoagressão. Evidenciam-se, assim, situações passíveis de intervenção e de elaboração de estratégias e políticas que possibilitem melhorar a qualidade de vida desses indivíduos e reduzir as taxas de comportamento suicida. 
Os achados deste estudo corroboram os apontamentos encontrados na literatura internacional acerca do comportamento suicida e as variáveis que podem estar relacionadas a esse fato. No entanto, este trabalho apresentou algumas limitações, como a necessidade do acompanhamento desses pacientes e a avaliação dos fatores de risco modificáveis após a avaliação desses pacientes no ambulatório transgênero, pois ele ainda é bem recente. Contudo, avaliações seguras de causa e efeito não puderam ser estabelecidas devido ao desenho inerente da pesquisa, necessitando, portanto, de estudos prospectivos em que a população transexual seja avaliada longitudinalmente e as variáveis possam ser mais bem investigadas no que tange às associações causais. Sugere-se ainda a realização de estudos prospectivos brasileiros, visto que a maior parte da literatura é estrangeira e o nosso país é um dos países com maiores taxas de homicídios entre a população transgênero.

\section{CONTRIBUIÇÕES INDIVIDUAIS}

Fábio Henrique Mendonça Corrêa - Contribuiu substancialmente para a concepção e design ou análise e interpretação dos dados; contribuiu substancialmente para redigir o artigo ou revisá-lo criticamente para conteúdo intelectual importante; deu a aprovação final da versão a ser publicada.

Bráulio Brandão Rodrigues - Contribuiu substancialmente para a análise e interpretação dos dados; contribuiu substancialmente para redigir o artigo ou revisá-lo criticamente para conteúdo intelectual importante.

Jussane Cabral Mendonça - Contribuiu substancialmente para revisá-lo criticamente para conteúdo intelectual importante; deu a aprovação final da versão a ser publicada.

Leonardo Rodrigues da Cruz - Contribuiu substancialmente para revisá-lo criticamente para conteúdo intelectual importante; deu a aprovação final da versão a ser publicada.

\section{CONFLITO DE INTERESSES}

Os Drs. Fábio Henrique Mendonça Corrêa, Bráulio Brandão Rodrigues, Jussane Cabral Mendonça e Leonardo Rodrigues da Cruz declaram não possuir conflito de interesses a serem relatados.

\section{AGRADECIMENTOS}

Gostaria de agradecer a todos que participaram da elaboração deste estudo (direta ou indiretamente), em especial ao Hospital São Vicente de Paula pela concessão do seu espaço físico e apoio técnico para a realização desta pesquisa.

\section{REFERÊNCIAS}

1. Cruz MHS. Refletindo sobre a diversidade de gênero no campo da educação. Saberes em Perspectiva. 2012:2 (2):13-32

2. Martins CF. Gênero e Sexualidade na Educação Contemporânea. Id on Line Revista Multidisciplinar e de Psicologia. 2017;10(33):257-70

3. Goularth NDR. Diversidade de gêneros e ensino de biologia: casos de prazeres e corporeidade não binários [monografia]. Seropédica: Universidade Federal Rural do Rio de Janeiro; 2015.

4. Rodrigues C. BUTLER, Judith P. Problemas de gênero: feminismo e subversão da identidade. Rev Estud Fem. 2005;13(1):179.

5. Segato RL. Os percursos do gênero na antropologia e para além dela (no 236). Brasília: Departamento de Antropologia/Universidade de Braślia; 1998.

6. Fausto-Sterling A. Cuerpos sexuados: la política de género y la construcción de la sexualidade. Barcelona: Melusina; 2006. 526p

7. Santos MA, de Souza RS, da Silva Lara LA, de Oliveira WA, Alexandre V, de Oliveira-Cardoso ÉA. Transexualidade, ordem médica e política de saúde: controle normativo do processo transexualizador no Brasil. Estudos Interdisciplinares em Psicologia. 2019;10(1):3-19.

8. Costa MB, Cândido JP, Bizerra PL. Acolhimento no âmbito da saúde pública sob a ética de transexuais. Cadernos de Educação, Saúde e Fisioterapia. 2017;4(8).

9. Zeluf G, Dhejne C, Orre C, Mannheimer LN, Deogan C, Höijer J, et al. Targeted victimization and suicidality among trans people: A web-based survey. LGBT Health. 2018;5(3):180-90.

10. Perez-Brumer A, Hatzenbuehler ML, Oldenburg CE, Bockting W. Individual-and structural-level risk factors for suicide attempts among transgender adults. Behav Med. 2015:41(3):164-71.

11. Distefano AS. Suicidality and self-harm among sexual minorities in Japan. Qual Health Res. 2008:18(10):1429-41.

12. Miller LR, Grollman EA. The social costs of gender nonconformity for transgender adults: Implications for discrimination and health. Sociol Forum (Randolph N J). 2015;30(3): $809-31$

13. Camino L, Silva PD, Machado A, Pereira C. A face oculta do racismo no Brasil: Uma análise psicossociológica. Rev Psicol Política. 2001;1(1):13-36.

14. Reisner SL, Vetters R, Leclerc M, Zaslow S, Wolfrum S, Shumer D, et al. Mental health of transgender youth in care at an adolescent urban community health center: a matched retrospective cohort study. J Adolesc Health. 2015;56(3):274-9.

15. Garcia-Vega E, Camero A, Fernandez M, Villaverde A. Suicidal ideation and suicide attempts in persons with gender dysphoria. Psicothema. 2018:30(3):283-8.

16. Marshall BD, Socías ME, Kerr T, Zalazar V, Sued 0, Arístegui I. Prevalence and correlates of lifetime suicide attempts among transgender persons in Argentina. J Homosex. 2016;63(7):955-67.

17. Grant J, Mottet L, Tanis J, Herman JL, Harrison J, Keisling M. National transgender discrimination survey report on health and health care. Washington, DC: National Center for Transgender Equality; 2010.

18. Lehavot K, Simpson TL, Shipherd JC. Factors associated with suicidality among a national sample of transgender veterans. Suicide Life Threat Behav. 2016:46(5):507-24.

19. Virupaksha HG, Muralidhar D, Ramakrishna J. Suicide and suicidal behavior among transgender persons. Indian J Psychol Med. 2016;38(6):505-9.

20. Crosby AE, Han B, Ortega LA, Parks SE, Gfroerer J; Centers for Disease Control and Prevention (CDC). Suicidal thoughts and behaviors among adults aged $\geq 18$ years - United States, 2008-2009. MMWR Surveill Summ. 2011;60(13):1-22.

21. da Cunha LR. Processo transgenitalizador: estado civil, necessidade de anuência e dever de informação da condição de transgênero. Direito UNIFACS - Debate Virtual. 2019;223.

22. Bezerra DS, Bezerra AK, de Souza RCM, Nogueira WBAG, Bonzi ARB, da Costa LMM. Homens transexuais: invisibilidade social e saúde mental. Temas em Saúde. 2018;18(1):428-44.

23. Hottes TS, Bogaert L, Rhodes AE, Brennan DJ, Gesink D. Lifetime prevalence of suicide attempts among sexual minority adults by study sampling strategies: a systematic review and meta-analysis. Am J Public Health. 2016;106(5):e1-12.

24. Zanello V. Saúde mental, gênero e dispositivos: cultura e processos de subjetivação. Curitiba: Appris; 2018 
25. Albuquerque GA, Figueiredo FWS, Paiva LS, Araújo MFM, Maciel ES, Adami F. Violencia y consumo de drogas está asociado con el suicidio en lesbianas, gays, bisexuales, transvestitas y transexuales: estudio transversal. Salud Ment. 2018;41(3):131-8.

26. Lian Q, Zuo X, Lou C, Gao E, Cheng Y. Sexual orientation and risk factors for suicidal ideation and suicide attempts: a multi-centre cross-sectional study in three Asian cities. J Epidemiol. 2015;25(2):155-61.

27. Yüksel §̧, Aslantaş Ertekin B, Öztürk M, Bikmaz PS, Oğlağu Z. A clinically neglected topic: Risk of suicide in transgender individuals. Noro Psikiyatr Ars. 2017;54(1):28-32.
28. Taliaferro LA, Muehlenkamp JJ. Nonsuicidal self-injury and suicidality among sexual minority youth: risk factors and protective connectedness factors. Acad Pediatr. 2017;17(7):715-22.

29. Bauer GR, Scheim Al, Pyne J, Travers R, Hammond R. Intervenable factors associated with suicide risk in transgender persons: a respondent driven sampling study in Ontario, Canada. BMC Public Health. 2015;15(1):525.

30. Paul E, Tsypes A, Eidlitz L, Ernhout C, Whitlock J. Frequency and functions of non-suicidal selfinjury: Associations with suicidal thoughts and behaviors. Psychiatry Res. 2015;225(3):276-82. 